\title{
Adaptação transcultural da versão brasileira da escala Social Rhythm Metric-17 (SRM-17) para a população angolana
}

\author{
Cross-cultural adaptation of the Brazilian version of the Social Rhythm Metric-17 (SRM-17) \\ for the population of Angola
}

\author{
Regina Lopes Schimitt ${ }^{1}$, Idalina Cesaltina Ribeiro Benedito ${ }^{2}$, Bruna Cláudia da Rocha ${ }^{2}$, Joyce Iracema \\ Angelo Chivia ${ }^{2}$, Maria Paz Loayza Hidalgo ${ }^{3}$
}

${ }^{1}$ Mestre e doutoranda, Programa de Pós-Graduação em Ciências Médicas, Faculdade de Medicina, Universidade Federal do Rio Grande do Sul (UFRGS), Porto Alegre, RS. ${ }^{2}$ Acadêmica de Medicina, Universidade Agostinho Neto, Luanda, Angola. ${ }^{3}$ Doutora. Professora adjunta, Departamento de Psiquiatria e Medicina Legal, Faculdade de Medicina, UFRGS.

Suporte financeiro: Fundo de Incentivo à Pesquisa e Eventos do Hospital de Clínicas de Porto Alegre (FIPE-HCPA).

\section{Resumo}

Introdução: O ritmo social é um conceito que integra a relação entre Zeitgebers (sincronizadores) sociais e os marcadores de tempo endógenos, e pode ser avaliado com a Escala de Ritmo Social (Social Rhythm Metric-17, SRM-17). O objetivo deste estudo foi realizar a adaptação da versão brasileira da SRM-17 para o português angolano, comparando as duas escalas em populações que utilizam o mesmo idioma mas apresentam diferenças culturais. Métodos: A versão brasileira da SRM-17 foi submetida à avaliação de 10 estudantes universitários angolanos, que analisaram o grau de clareza de cada um dos 15 itens do instrumento usando uma escala visual analógica de $10 \mathrm{~cm}$ e propuseram modificações ao texto. Foi realizada revisão dos resultados para a elaboração da versão final, bem como prova de leitura e relatório final.

Resultados: A versão final angolana manteve uma equivalência de itens com relação à versão em português brasileiro. A versão avaliada demonstrou um grau satisfatório de clareza e equivalência semântica na maioria dos itens. Porém, alguns itens apresentaram um escore na clareza inferior à média aritmética de compreensão global do instrumento $(8,38 \pm 1,0)$.

Conclusão: Apesar de o português ser o idioma oficial nos dois países, há diferenças culturais significativas nas duas populações. Este trabalho apresenta uma versão adaptada à realidade angolana de um instrumento específico para aferir ritmo social. O processo de adaptação transcultural deve efetivar-se com estudos de validação do instrumento final em uma amostra maior da população, onde também poderão ser avaliadas as equivalências operacional, de medida e funcional.

Descritores: Social Rhythm Metric, Zeitgeber, cronobiologia, ritmo biológico.

\begin{abstract}
Introduction: Social rhythm is a concept that correlates social Zeitgebers (synchronizers) with endogenous markers of time, and can be assessed with the Social Rhythm Metric-17 (SRM-17). The aim of this study was to adapt the Brazilian version of the SRM-17 to Angolan Portuguese, comparing the two scales in samples that speak the same language but have cultural differences.

Methods: The Brazilian version of the SRM-17 was assessed by 10 Angolan students who analyzed the intelligibility of the 15 sentences contained in the instrument using a 10-cm visual analog scale and proposed changes to the text. Results were reviewed for the generation of a final Angolan version, followed by a reading test and the production of a final report.

Results: The final Angolan version of SRM-17 maintained an equivalence of items in relation to the Brazilian Portuguese version. The version assessed by the students showed a satisfactory degree of intelligibility and semantic equivalence in most items. However, some items presented intelligibility results below the mean total scores of the scale $(8.38 \pm 1.0)$.

Conclusion: Although the populations of Brazil and Angola speak the same language, significant cultural differences were found between the two countries. This paper presents an instrument to assess social rhythm adapted to the Angolan culture. The cross-cultural adaptation process herein described should be continued by validating the final version of the instrument in a larger sample and by assessing operational, functional, and measure equivalence.
\end{abstract}

Keywords: Social Rhythm Metric, social Zeitgeber, chronobiology, biologic rhythm.

Correspondência:

Maria Paz Loayza Hidalgo, Laboratório de Cronobiologia, HCPA/UFRGS, Rua Ramiro Barcelos, 2350/2201, CEP 90035-903, Porto Alegre, RS, Brasil. E-mail: 00072803@ ufrgs.br,mpaz@cpovo.net

Não foram declarados conflitos de interesse associados à publicação deste artigo.

Copyright $(\odot$ Revista de Psiquiatria do Rio Grande do Sul - APRS

Recebido em 10/03/2010. Aceito em 26/04/2010. 


\section{Introdução}

O ritmo social é um conceito que integra a relação entre fatores sociais e marcadores de tempo endógenos. Os sincronizadores exógenos, como alimentação, exercício e trabalho, são também denominados de Zeitgebers, termo alemão cunhado por Aschoff nos anos 1970 que significa doadores de tempo ${ }^{1}$. As pistas sociais, atividades temporalmente organizadas em torno de interações sociais, constituem-se em Zeitgebers sociais. Essas pistas interferem na expressão dos ritmos biológicos, pois têm o intuito de adaptar a função dos sistemas fisiológicos ao meio externo. A compreensão dessa interação tem sido alvo de áreas voltadas ao estudo do comportamento, como a neurociência, a psiquiatria e a psicologia. Para possibilitar o estudo dos Zeitgebers sociais, têm sido elaborados instrumentos para aferir o ritmo social, como a Escala de Ritmo Social (Social Rhythm Metric-17, SRM-17).

A SRM-17 foi concebida em 1990 por pesquisadores da Universidade de Pittsburg para estudar a associação entre rupturas no ritmo social e o início de episódios de transtorno de humor ${ }^{2-6}$. No Brasil, foi validada por Schimitt \& Hidalgo ${ }^{7}$ e por Schimitt et al. ${ }^{8}$ em 2009 (Anexo 1).

$\mathrm{O}$ acordo de unificação da língua portuguesa, posto em prática a partir de 2009 pelos oito países lusófonos do mundo, prevê apenas a unificação da ortografia do idioma, desconsiderando, na prática, diferenças culturais que se erigem como barreiras à compreensão interlinguística, já que, linguisticamente, o que mais difere entre as diferentes versões da língua portuguesa são a sintaxe e o vocabulário, e não a ortografia. Sendo assim, para o desenvolvimento da pesquisa cronobiológica nos países de língua portuguesa no continente africano, foi necessário investigar a adequação da escala SRM-17 brasileira a essa realidade.

O presente estudo descreve o processo de adaptação da versão brasileira da SRM-17 ao português angolano, com ênfase na importância do estudo de validação de um instrumento para a população para a qual se deseja inferir os resultados, mesmo quando se tem como origem o mesmo idioma.

\section{Sujeitos e métodos}

A SRM-17 é um formulário autoaplicável que consiste em uma lista de 15 atividades genéricas fixas e duas opcionais para que o sujeito registre, ao final do dia, durante um determinado período de tempo, se essas atividades foram ou não realizadas, o horário em que foram realizadas e a quantidade e qualidade de interações sociais vivenciadas durante a realização das mesmas.

A escala apura, a partir de um algoritmo, dois índices mais importantes ${ }^{3-5}$ o Hit e o ALI. O Hit é um conceito para o qual não existe uma tradução satisfatória em português. Ele indica o índice de regularidade no estilo de vida de uma pessoa. Se uma atividade ocorre mais de três vezes por semana no mesmo horário ou dentro de uma faixa temporal que abrange os 45 minutos anteriores e posteriores a uma hora calculada como habitual, ela é considerada um Hit, ou seja, ela contribui de forma significativa com a regularidade. A quantidade de Hits representa a regularidade na vida de um indivíduo e é o índice mais importante da escala. O escore de regularidade não possui um ponto de corte, mas varia de um mínimo de 0 (nenhuma regularidade) até um máximo que depende do número de dias aferidos. Se, por exemplo, o período aferido compreende 7 dias, o escore máximo é 7; se for 9 dias, o escore máximo é 9 , etc. A escala foi concebida para avaliar a regularidade em um período mínimo de 1 semana. O índice ALI representa o volume de atividades diferentes realizadas no período, sendo calculado através da contagem simples de toda atividade realizada a cada dia. De acordo com Monk et al. ${ }^{6}$, um escore inferior a 59 inviabiliza a avaliação de regularidade. Portanto, o ALI pode variar de 59 (pouca atividade) até um máximo que depende do período aferido. Se, por exemplo, o período aferido compreende 7 dias e o sujeito se engaja em todas as 17 atividades contempladas pela escala, então o escore máximo será 119 (17 x 7).

Na versão angolana da SRM-17, buscou-se obter um instrumento equivalente à versão brasileira do ponto de vista semântico ${ }^{8}$. A equivalência semântica diz respeito ao quanto se conserva de significado na tradução para outra língua, desde os pontos de vista referencial (que diz respeito a ideias ou objetos a que uma palavra ou conjunto de palavra se referem), conotativo (resposta emocional evocada por uma palavra), estilístico ou social (que concerne ao quanto uma palavra é apropriada num contexto específico condicionado a geografia, tempo, estilo, nível de formalidade, sexo, etc., e imprópria em outro contexto), afetivo (modo como as palavras usadas podem refletir sentimentos e pontos de vista), refletido (modos como é possível interpretar uma palavra), colocativo (modo pelo qual uma palavra, extraída de um contexto estrangeiro, pode ganhar força ou significado extra) e temático (modo pelo qual um significado particular é dado a uma mensagem pelo modo como a frase se organiza $)^{9-23}$.

Para tanto, a versão brasileira da SRM-17 foi submetida à avaliação de 10 pesquisadores estudantes universitários provenientes de Angola. Esses 10 estudantes analisaram o grau de clareza de cada um dos 15 itens do instrumento por meio de uma escala visual analógica de $10 \mathrm{~cm}^{24,25}$. As instruções dadas aos estudantes são apresentadas a seguir:

Este material que você está começando a ler é a lista dos itens que compõem a versão brasileira da Escala de Ritmo Social. Neste momento, não é importante compreender os objetivos da Escala de Ritmo Social, e sim saber se as frases da versão brasileira são adequadas à compreensão de quem fala o português usado em Angola. Para isso, você precisa cumprir quatro etapas:

1. Leia atentamente cada frase. 
2. Descreva o quão clara está a questão, colocando um traço perpendicular à linha abaixo da questão. Lembre que 0 significa "nada claro" e 10 significa "absolutamente claro".

3. Descreva, abaixo de cada frase, o que você entendeu que a frase quer dizer.

Faça sugestões de modificação que tornem a frase mais adequada ao português falado em Angola. Você verá que muitas frases podem permanecer da mesma maneira, mas muitas ficariam melhor se fossem modificadas.

A compreensão global do instrumento foi definida como a média aritmética das 15 questões objetivas que compõem o instrumento. Foram definidos como indicadores de compreensão insuficientes valores abaixo da média de compreensão global do instrumento.

Os participantes também foram convidados a anotar críticas e sugestões, as quais foram levadas em consideração no estabelecimento da versão definitiva do instrumento. Por último, o instrumento passou por prova de revisão para a correção de possíveis erros que não tenham sido detectados em algum ponto do processo.

A partir da revisão dos resultados de avaliação de clareza, foi elaborada a versão final do instrumento, seguida de prova de leitura e relatório final.

\section{Resultados}

Neste trabalho, observamos que, apesar do idioma comum entre Brasil e Angola, o instrumento teve que sofrer modificações para permitir a compreensão das atividades na população angolana. Os avaliadores da escala detectaram a necessidade de modificação de alguns itens a fim de obter equivalência conceitual.

A Tabela 1 mostra que a versão avaliada demonstrou um grau satisfatório de clareza e equivalência semântica. Porém, alguns itens apresentaram uma média de clareza inferior à média aritmética total da escala $(8,38 \pm 1,0)$.

Tabela 1 - Versão avaliada e versão final da Escala de Ritmo Social com as respectivas médias e desvio padrão do grau de clareza de cada questão medido por escala visual analógica $(\mathrm{n}=10)$

\begin{tabular}{|c|c|c|}
\hline Versão avaliada & Versão final & Média \pm DP (cm) \\
\hline 1. Saí da cama & 1. Levantei-me da cama & $8,50 \pm 2,3$ \\
\hline $\begin{array}{l}\text { 2. Falei pela primeira vez com outra pessoa } \\
\text { (pessoalmente ou por telefone) }\end{array}$ & $\begin{array}{l}\text { 2. Falei pela primeira vez com outra pessoa } \\
\text { (pessoalmente ou por telefone) }\end{array}$ & $8,90 \pm 2,3$ \\
\hline $\begin{array}{l}\text { 3. Ouvi rádio ou assisti TV (em casa, no carro, no } \\
\text { trabalho, ou em outro lugar) }\end{array}$ & $\begin{array}{l}\text { 3. Ouvi rádio ou assisti TV (em casa, no carro, no } \\
\text { trabalho, ou em outro lugar) }\end{array}$ & $9,89 \pm 0,333$ \\
\hline 4. Tomei café da manhã & 4. Tomei o pequeno almoço (matabichei) & $9,40 \pm 1,897$ \\
\hline 5. Saí de casa & $\begin{array}{l}\text { 5. Sai de casa de pela primeira vez no dia (para } \\
\text { trabalho, escola, etc.) }\end{array}$ & $6,00 \pm 4,375^{*}$ \\
\hline 6. Fiz exercício físico & 6. Fiz exercício fisico (caminhada, ginástica, dança, etc.) & $9,50 \pm 1,581$ \\
\hline $\begin{array}{l}\text { 7. Comecei o trabalho (ou a escola, o trabalho de } \\
\text { casa, trabalho voluntário, cuidados com crianças } \\
\text { ou outros membros da família, etc.) }\end{array}$ & $\begin{array}{l}\text { 7. Comecei o trabalho diário (no emprego, na escola, } \\
\text { no trabalho de casa, trabalho voluntário, etc.) }\end{array}$ & $9,40 \pm 1,578$ \\
\hline 8. Almocei & 8. Almocei & $9,50 \pm 1,581$ \\
\hline 9. Tirei uma soneca à tarde & 9. Descansei à tarde & $7,70 \pm 3,889^{*}$ \\
\hline 10. Fiz um lanche & 10. Lanchei (comi ou bebi algo à tarde) & $8,80 \pm 2,098$ \\
\hline 11. Cheguei em casa pela última vez & $\begin{array}{l}\text { 11. Voltei para casa (do trabalho, escola, etc.) pela } \\
\text { última vez no dia }\end{array}$ & $3,25 \pm 4,400^{*}$ \\
\hline 12. Jantei & 12. Jantei & $9,50 \pm 1,581$ \\
\hline $\begin{array}{l}\text { 13. Fiz uma atividade de lazer (assisti TV, fui ao } \\
\text { cinema, li, etc.) }\end{array}$ & $\begin{array}{l}\text { 13. Fiz uma atividade de lazer (assisti TV, fui ao } \\
\text { cinema, li, etc.) }\end{array}$ & $9,00 \pm 1,633$ \\
\hline 14. Desliguei a TV ou o rádio pela última vez & 14. Desliguei a TV ou o rádio pela última vez (no dia) & $7,80 \pm 3,824^{*}$ \\
\hline 15. Fui para a cama & 15. Fui me deitar & $9,80 \pm 0,632$ \\
\hline Escore total & & $8,3778 \pm 0,99926$ \\
\hline
\end{tabular}

$\mathrm{DP}=$ desvio padrão.

*Itens com indicadores de compreensão insuficientes (valores abaixo da média global do instrumento). 
Seguem abaixo algumas considerações e a descrição das modificações realizadas a partir dos procedimentos de avaliação de equivalência conceitual, de item e semântica:

1. Foi mantido o tempo verbal. Os verbos continuaram conjugados no pretérito perfeito, porque a escala deverá ser preenchida ao final do dia, quando já ocorreram as ações. Isso permite a quem está respondendo organizar-se temporalmente.

2. Os verbos foram mantidos em todas as frases para manter a coerência entre os itens.

3. O item 1, "Saí da cama", foi modificado para "Levantei-me da cama", pois em Angola, sair da cama não tem a conotação de acordar e se levantar.

4. Os itens 2 e 3 foram mantidos conforme a versão brasileira.

5. O item 4, "Tomei café da manhã", não é usual em Angola, portanto foi modificado para "Tomei o pequeno almoço (matabichei)", por ser esta forma considerada mais adequada à realidade angolana.

6. O item 5, "Saí de casa", foi modificado para "Saí de casa de pela primeira vez no dia (para trabalho, escola, etc.)", pois estava sendo confundido com mudança de endereço.

7. O item 6, "Fiz exercício físico (caminhada, ginástica, dança, etc.)" foi mantido conforme a versão brasileira. Foram, porém, acrescentados exemplos de atividades, de acordo com sugestões de alguns dos estudantes.

8. O item 7, "Comecei o trabalho (ou a escola, o trabalho de casa, trabalho voluntário, cuidados com crianças ou outros membros da família, etc.)", foi modificado para "Comecei o trabalho diário (no emprego, na escola, no trabalho de casa, trabalho voluntário, etc.)", pois houve confusão com começar num primeiro emprego.

9. O item 8, "Almocei", foi mantido desta forma, como na versão brasileira.

10. O item 9, "Tirei uma soneca à tarde", foi modificado para a forma mais usual "Descansei à tarde".

11. No item 10, a expressão "Fiz um lanche" foi substituída por "Lanchei (comi ou bebi algo à tarde)", de forma semelhante à versão em inglês "Have an evening snack/drink".

12. O item 11, "Cheguei em casa pela última vez", foi modificado para "Voltei para casa (do trabalho, escola, etc.) pela última vez no dia". Os avaliadores compreenderam uma finitude de existência associada à versão brasileira do item ("pela última vez retornei para casa e depois faleci"), ou seja, não era compreendido o fato de o indivíduo retornar de uma atividade rotineira para o lar.

13. Os itens 12 e 13 foram mantidos como na versão brasileira.

14. O item 14, "Desliguei a TV ou o rádio pela última vez" foi também modificado para "Desliguei TV ou o rádio pela última vez (no dia)", porque novamente os avaliadores assinalaram a ideia de finitude.

15. O item 15, "Fui para a cama", foi modificado para "Fui me deitar", opção mais adequada à cultura angolana.

O Anexo 2 apresenta a versão final da escala adaptada para a população angolana.

\section{Discussão}

Este estudo demonstra que, mesmo sendo o português o idioma oficial adotado nos dois continentes avaliados, diferenças significativas foram encontradas no entendimento de cada questão, salientando a importância de estudos de validação de instrumentos para uma adequada aferição do construto. Apesar das tentativas governamentais de uniformização do idioma português, as questões culturais e históricas se impõem no nível semântico e sintático, como foi possível observar na questão 4 , em que a forma "Tomei café da manhã" da escala brasileira foi melhor representada pela expressão "matabichei" na versão angolana.

Além disso, algumas questões foram julgadas pelos avaliadores como demonstrando clareza adequada; porém, ao serem questionados sobre o que entendiam da pergunta, constatou-se que o sentido compreendido não era o que se desejava aferir, como, por exemplo, no item 11, "Cheguei em casa pela última vez", na versão brasileira, que teve que ser modificado pois era compreendido como "morri" pelos participantes do estudo angolano.

A padronização de instrumentos e métodos é um recurso que otimiza as ações em pesquisa e permite o intercâmbio de conhecimentos na comunidade científica mundial ${ }^{9,17}$. Porém, na realização de estudos comparativos entre culturas diferentes, fatores socioculturais específicos devem ser levados em consideração, sobretudo quando essas culturas compartilham o mesmo idioma oficial. Idioma é um termo referente à língua usada para identificar uma nação em relação às demais e está relacionado à existência de um estado político, ou seja, sempre vinculado à língua oficial de um país. $\mathrm{O}$ fato de duas culturas diferentes compartilharem o mesmo idioma oficial pode ser um complicador na medida em que se pressuponha que as duas culturas façam o mesmo uso do idioma. As diferenças observadas no uso do idioma no presente estudo aproximam-se do conceito de dialeto, na medida em que são variedades linguísticas regionais e sociais, diferenças não erradicadas pelo acordo ortográfico.

Este estudo demonstra que, no campo da investigação científica, uma imprecisão dessa magnitude, resultante da pressuposição de que o acordo ortográfico pode transpor barreiras culturais, pode conduzir a erros nos dados obtidos quando não se considera a adaptação do instrumento à cultura dos sujeitos investigados. Este estudo vai ao encontro do questionamento sobre os limites das áreas da ciência, pois se insere na interface entre diversas áreas de investigação, a saber, antropologia, linguística e ciência do comportamento. 
A partir da adaptação da escala, será possível aprofundar e ampliar o estudo da cronobiologia no continente africano. Num sentido mais amplo, dessa linha de pesquisa emergem questionamentos sobre os efeitos do desacoplamento entre a organização temporal da sociedade e os ritmos biológicos individuais, revelando o impacto dessa incompatibilidade sobre a qualidade de vida das pessoas ${ }^{25}$.

Estudos comparativos entre realidades geográficas diferentes põem em evidência o papel dos fatores ambientais na expressão de genes relacionados ao relógio biológico ${ }^{26}$, fatores esses que abrangem os Zeitgebers sociais. Existem estudos que relacionam a regularidade do ritmo social com maior rapidez de recuperação de episódios de transtornos afetivos, bem como com a existência de um maior intervalo entre um episódio e outro ${ }^{4}$. Assim, a investigação do ritmo social interessa, sobretudo, ao estudo da prevenção de doenças decorrentes da dessincronização de ritmos biológicos e propõe uma quebra de paradigma na forma como a sociedade se estrutura temporalmente ${ }^{27}$.

Futuros estudos devem contribuir para o desenvolvimento de instrumentos de aferição que permitam reproduzir pesquisas em diferentes sociedades, possibilitando aferir o efeito Zeitgeber social e sua interação com o Zeitgeber fótico, bem como as relações destes com a clínica ${ }^{2,3,6}$ e com a performance em atividades de estudo e trabalho ${ }^{28}$, e ainda as relações destes com envelhecimento ${ }^{29}$.

\section{Conclusões}

Este trabalho apresenta uma versão adaptada ao português angolano de um instrumento específico para aferir ritmo social, equivalente a versão original da escala em português brasileiro dos pontos de vista conceitual, de item e semântico. As diferenças continentais evidenciadas neste estudo enfatizam a importância de estudos de adaptação transcultural de instrumentos, mesmo quando se considera o mesmo idioma. O estudo de validação do instrumento final em uma amostra maior da população poderá avaliar as equivalências operacional, de medida e funcional, bem como trazer importantes dados sobre a influência social na regulação do sistema temporizador em Angola.

\section{Agradecimentos}

Ao Dr. Thimoty Monk, da Universidade de Pittsburg, criador da Escala de Ritmo Social, e ao Prof. Luiz MennaBarreto, do Grupo de Cronobiologia Humana da Universidade de São Paulo, pelas aulas ministradas ao Grupo de Cronobiologia Humana do Hospital de Clínicas de Porto Alegre.

\section{Referências}

1. Aschoff J, Fatranská M, Giedke H. Human circadian rhythms in continuous darkness: entrainment by social cues. Science. 1971;171:213-5.

2. Monk TH, Flaherty JF, Frank E, Hoskinson K, Kupfer DJ. The Social Rhythm Metric: an instrument to quantify the daily rhythms of life. J Nerv Ment Dis. 1990;178:120-6.

3. Monk TH, Kupfer DJ, Frank E, Ritenour AM. The Social Rhythm Metric (SRM): measuring daily social rhythms over 12 weeks. Psychiatry Res. 1990;36:195-207.

4. Frank E, Swartz HA, Kupfer DJ. Interpersonal and social rhythm therapy: managing the chaos of bipolar disorder. Biol Psychiatry. 2000;48:593-604

5. Monk TH, Kupfer DJ, Frank E, Potts JM, Kupfer DJ. A simple way to measure daily lifestyle regularity. J Sleep Res. 2002;11:183-90.

6. Monk TH, Petrie SR, Hayes AJ, Kupfer DJ. Regularity of daily life in relation to personality, age, gender, sleep quality and circadian rhythms. J Sleep Res. 1994;3:196-205.

7. Schimitt R, Hidalgo MP. Tradução para o português e validação de conteúdo da versão em português da Social Rhythm Metric-17 (SRM-17). Rev Psiquiatr Rio Gd Sul. 2009;31:120-7.

8. Schimitt RL, Zanetti T, Mayer M, Koplin C, Guarienti F, Hidalgo MP. Propriedades psicométricas da Escala de Ritmo Social em trabalhadores de turno regular. Rev Bras Psiquiatr. 2010;32:47-55.

9. Moraes CL, Hasselmann MH, Reichenheim ME. Adaptação transcultural para o português do instrumento Revised Conflict Tactics Scales (CTS2) utilizado para identificar violência entre casais. Cad Saude Publica. 2002;18:167-76.

10. Wild D, Grove A, Martin M, Eremenco S, McElroy S, Verjee-Lorenz A, et al. Principles of good practice for the translation and cultural adaptation process for Patient-Reported Outcomes (PRO) measures: report of the ISPOR Task Force for Translation and Cultural Adaptation. Value Health. 2005;8(2):94-104.

11. Hauck S, Schestatsky S, Terra L, Knijnik L, Sanchez P, Ceitlin LH. Adaptação transcultural para o português brasileiro do Parental Bonding Instrument (PBI). Rev Psiquiatr Rio Gd Sul. 2006;28(2):61-8.

12. Herdman M, Fox-Rushby J, Badia X. A model of equivalence in the cultural adaptation of HRQOL instruments: the universalist approach. Qual Life Res. 1998;7:323-35.

13. Beaton DE, Bombardier C, Guillemin F, Ferraz MB. Guidelines for the process of cross-cultural adaptation of Self-Report Measures. Spine. 2000;24:3186-91.

14. Chang AM, Chau JP, Holroyd E. Translation of questionnaires and issues of equivalence. J Adv Nurs. 1999;29(2):316-22.

15. Fiszman A, Cabizuca M, Lanfredi C, Figueira I. A adaptação transcultural para o português do instrumento Dissociative Experiences Scale para rastrear e quantificar os fenômenos dissociativos. Rev Bras Psiquiatr. 2004;26(3):164-73.

16. Reichenheim ME, Moraes CL. Operacionalização de adaptação transcultural de instrumentos de aferição usados em epidemiologia. Rev Saude Publica, 2007;41(4):665-73.

17. Jorge Miguel R. Adaptação transcultural de instrumentos de pesquisa em saúde mental. Rev Psiquiatr Clin. 1998;25(5):233-9.

18. Hasselmann MH, Reichenheim ME. Adaptação transcultural da versão em português da Conflict Tactics Scales Form R (CTS-1), usada para aferir violência no casal: equivalências semântica e de mensuração. Cad Saude Publica. 2003;19(4):1083-93.

19. Eremenco SL, Cella D, Arnold BJ. A comprehensive method for the translation and cross-cultural validation of health status questionnaires. Eval Health Prof 2005;28(2):212-32.

20. Wang W-L, Lee H-L, Fetzer SJ. Challenges and strategies of instrument translation. West J Nurs Res. 2006;28(3):310-20.

21. Sperber AD. Translation and validation of study instruments for cross-cultural research. Gastroenterology. 2004;126:124-8.

22. Harb A, Caumo W, Hidalgo MP. Tradução e adaptação da versão brasileira do Night Eating Questionnaire (NEQ). Cad Saude Publica. 2008;24(6):1368-76.

23. Fachel JMG, Camey S. Avaliação psicométrica: a qualidade das medidas e o entendimento dos dados. In: Cunha JA. Psicodiagnóstico V. $5^{\text {a }}$ ed. Porto Alegre: Artmed; 2000. p. 158-70.

24. Clark P, Levielle P, Martinez H. Learning from pain scales: patient perspective. J Rheumatol. 2003;30:1584-8.

25. Creamer P, Lethbridge-Cejku M, Hochberg MC. Determinants of pain severity in knee osteoarthritis: effect of demographic and psychosocial variables using three pain measures. J Rheumatol. 1999;26:1785-92.

26. Allebrandt KV, Teder-Laving M, Akyol M, Pichler I, Müller-Myhsok B, Pramstaller P, et al. CLOCK gene variants associate with sleep duration in two independent populations. Biol Psychiatry. 2010;67(11):1040-7.

27. Schimitt RL, Hidalgo MP, Caumo W. Ritmo social e suas formas de mensuração: uma perspectiva histórica. Rev Estud Pesq Psicol. 2010;10(2):457-70.

28. Monk TH. What can the chronobiologist do to help the shift worker? J Biol Rhythms. 2000;15(2):86-94.

29. Monk TH, Reynolds III CF, Kupfer DJ, Hoch CC, Carrier J, Houck R. Differences over the life span in daily life-style regularity. Chronobiol Int. 1997;14(3):295-306. 


\section{Anexo 1 - Escala de Ritmo Social (versão brasileira)}

Universidade Federal do Rio Grande do Sul - Grupo de Pesquisa em Cronofarmacologia da Dor e do Comportamento

ESCALA DE RITMO SOCIAL - BRASIL

Por favor, preencha esta tabela no fim do dia.

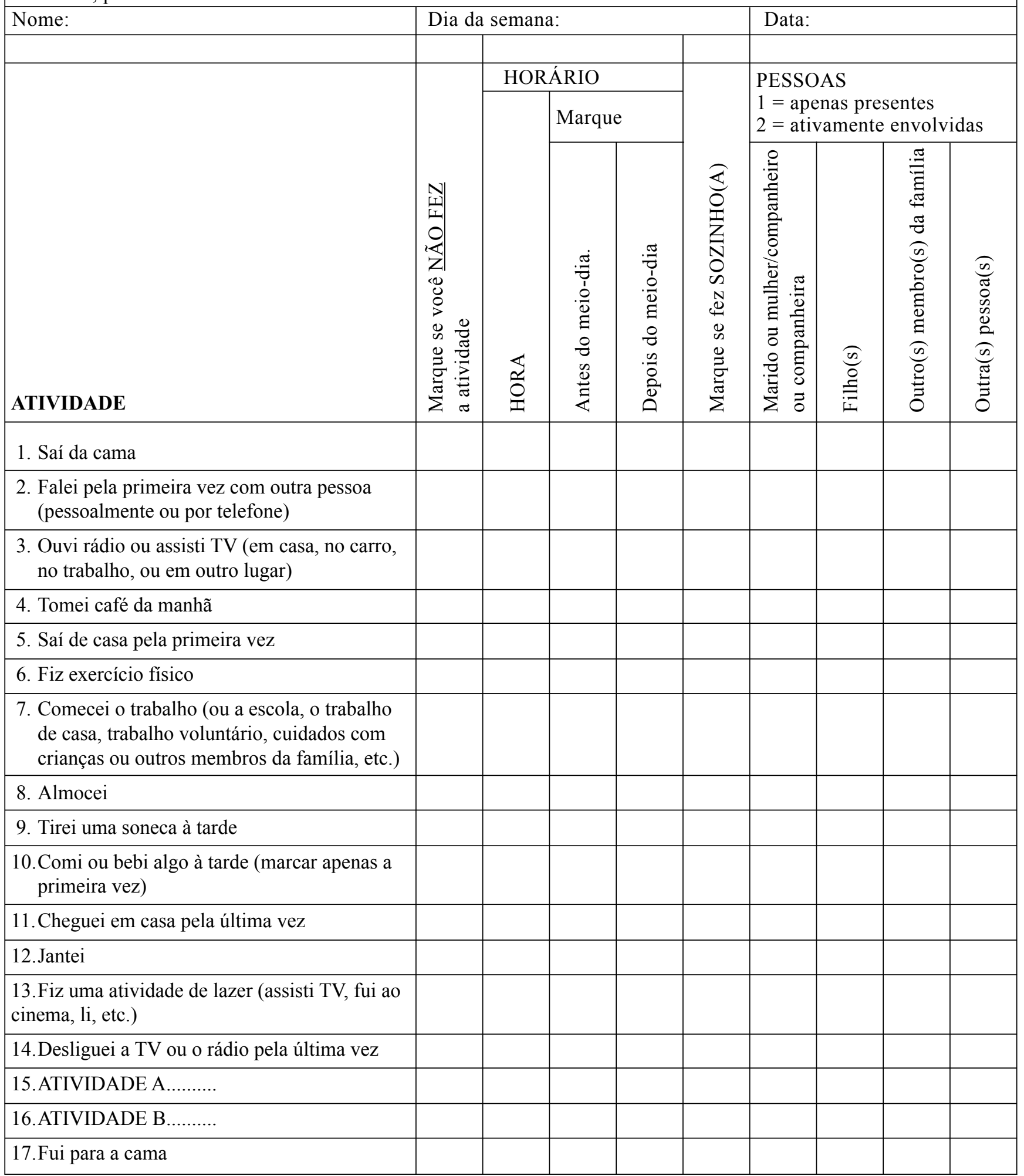




\section{Anexo 2 - Escala de Ritmo Social (versão angolana)}

Universidade Federal do Rio Grande do Sul - Laboratório de Cronobiologia/HCPA

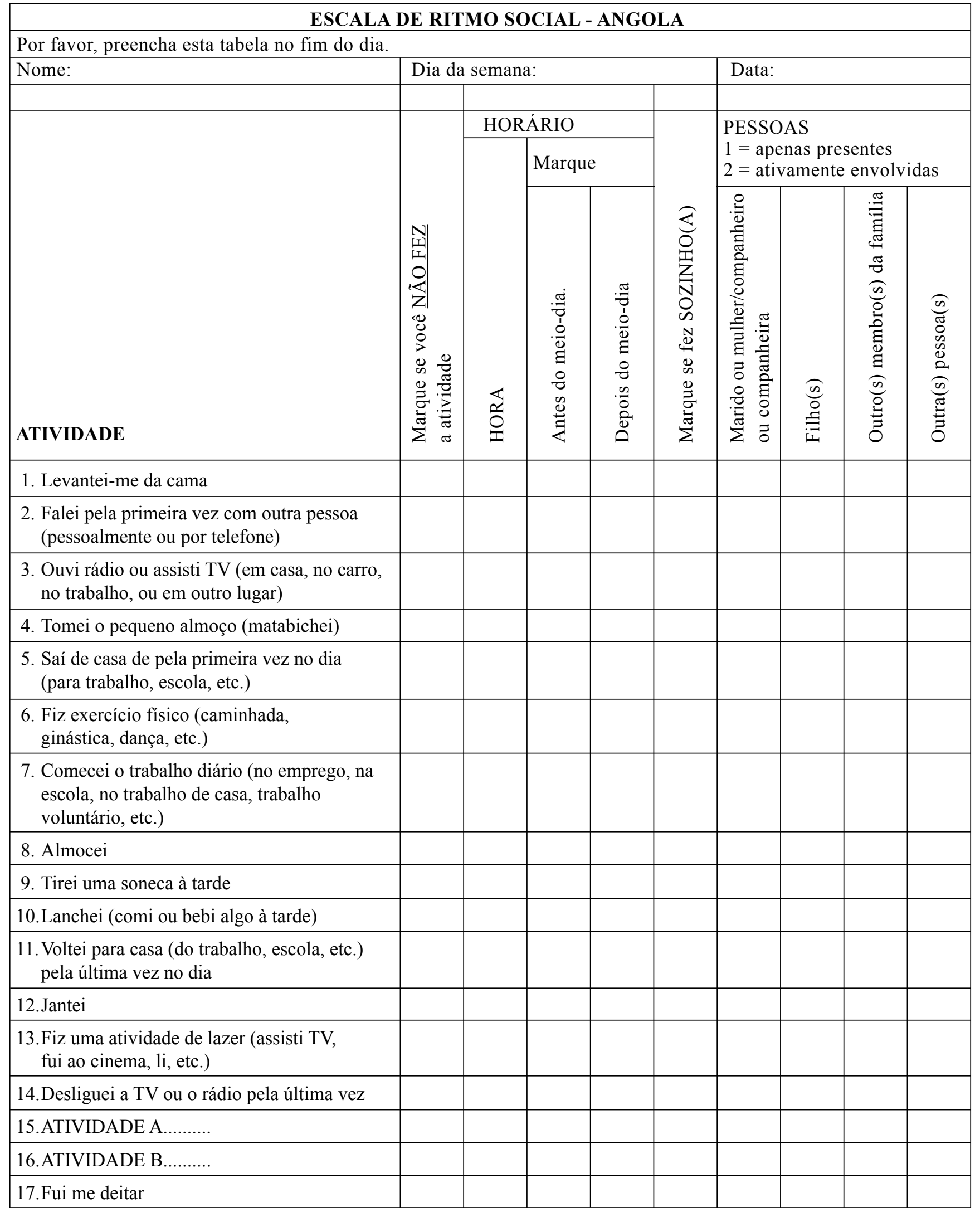

